\title{
STUDY OF DRUG UTILIZATION PATTERN OF CALCIUM, VITAMIN D SUPPLEMENTS, AND ANTI-OSTEOPOROSIS DRUGS IN PATIENTS WITH OSTEOPOROTIC FRACTURE: A PROSPECTIVE CROSS-SECTIONAL STUDY
}

\author{
ANGEL MARIA AUGUSTINE, DESSAISNEHA SHAMRAO, SUJAN BASNET*, ANCHAN NAMITA BHOJA, JAVEDH SHAREEF \\ Department of Pharmacy Practice, NGSM Institute of Pharmaceutical Sciences, Deralakatte, Mangaluru - 575 018, Karnataka, India. \\ Email: sujanbasnetchettri@gmail.com
}

Received: 2 June 2018, Revised and Accepted: 09 August 2018

\section{ABSTRACT}

Objective: This study was carried out to assess the drug utilization pattern of calcium, Vitamin D supplements, and anti-osteoporosis drugs in patients with osteoporotic fracture in an Indian teaching hospital.

Methods: A cross-sectional study was carried out for 6 months in outpatients receiving treatment from the orthopedic department of an Indian teaching hospital. Demographic details, comorbidities, types of fracture, laboratory parameters, and drug therapy were collected from the patient case records who met the study criteria and documented in the data collection form. All the collected details were later analyzed using descriptive statistics.

Results: A total of 73 patient's prescriptions were reviewed, in which $45.2 \%$ were male and $54.8 \%$ were female. Majority of the patients were in the age group of 50-60 years with mean age of $63.52 \pm 11.06$ years. Hypertension $(27.16 \%)$ was the highest comorbidity identified in the study followed by diabetes (18.51\%) and coronary artery disease (14.81\%). Of the total patients, $65.75 \%$ were prescribed with calcium and Vitamin D supplements and $2.73 \%$ received bisphosphonates.

Conclusion: The study shows the pattern of drug use in elderly with osteoporotic fractures. Since calcium and Vitamin D are vital for treating osteoporotic fractures, adequate monitoring of serum levels of calcium and Vitamin D3 may help to prescribe doses with desired need and safety level.

Keywords: Osteoporotic fractures, Calcium, Vitamin D supplements, Prescribing pattern.

(C) 2018 The Authors. Published by Innovare Academic Sciences Pvt Ltd. This is an open access article under the CC BY license (http://creativecommons. org/licenses/by/4. 0/) DOI: http://dx.doi.org/10.22159/ajpcr.2018.v11i12.27666

\section{INTRODUCTION}

Osteoporosis is a major health concern worldwide among the aging individuals that has a remarkable financial impact on the society. Osteoporosis, meaning "porous bone," is a skeletal disorder characterized by compromised bone strength with decreased bone mass and deterioration of bone microarchitecture, leading to increased bone fragility, predisposing a person to increased risk of fracture [1]. According to the World Health Organization criteria, osteoporosis is defined as a bone mineral density (BMD) that lies 2.5 standard deviations (SDs) or more below the average value for young person (T-score of <-2.5 SD) [2]. Fractures associated with osteoporosis have a significant impact on patients' quality of life and health-care costs, leading to increased risk for morbidity and mortality. The major risk factors that may lead to fractures are age, decreased BMD, low body mass index, a history of a prior fracture at a site characteristic for osteoporosis, a parental history of hip fracture, smoking, alcohol use, glucocorticoid use, and rheumatoid arthritis. Due to menopause, after the age of 50-55 years, women are more prone to fractures. Men are also at 25\% fracture risk after they cross the age of 50 years $[3,4]$.

General fracture prevention strategies include an increase in dietary calcium intake, a higher level of physical activities at all ages, reduction in the prevalence of smoking, decrease in alcohol consumption, fall prevention program, and hip protectors. The current management on osteoporosis includes comprehensive education for lifestyle modification, supplementation of calcium and Vitamin D, and antiosteoporosis drugs such as anti-resorptive and bone formation agents [5].
Calcium and Vitamin D supplements play a major role in treating osteoporosis and can reduce the risk of fracture. A study findings support a role for calcium and Vitamin D supplementation in osteoporosis treatment, as the diets of many osteoporotic patients are deficient in one or both. It has also been identified that there is a reduction in risk of fractures when calcium and Vitamin D are given in combination rather than Vitamin D alone [6].

In many countries, the predominant source of calcium and Vitamin D are milk, yogurt, cheese, orange juice, and dietary supplements which have a positive effect on bone loss and risk of fracture. A study conducted by National Osteoporosis Foundation in 120 elderly patients showed that the mean total daily calcium intake was $970 \mathrm{mg}$ which included $686 \mathrm{mg}$ through milk, yogurt, and cheese along with supplementation of $284 \mathrm{mg} /$ day $[7,8]$.

A better understanding of this process is possible with the study of drug utilization pattern of calcium, Vitamin D, and anti-osteoporosis drugs in patients with osteoporotic fractures. Hence, the study was carried out with the objective to assess the drug utilization pattern of calcium, Vitamin D supplements, and anti-osteoporosis drugs in patients with osteoporotic fracture visiting the orthopedic outpatient department in a tertiary care teaching hospital.

\section{MATERIALS AND METHODS}

A prospective cross-sectional study was conducted in outpatient orthopedics department of a tertiary care teaching hospital located in Dakshina Kannada, Deralakatte, Mangaluru. The study was carried out for 6 months (October 2016-March 2017). This study was approved by the Institutional Ethics Committee (REF: INST.EC/EC/112/2016- 
2017). A total of 73 patients of either gender aged 50 years or above diagnosed with osteoporotic fracture receiving treatment in the outpatient orthopedics department were included in the study. Hospitalized orthopedic patients and patients visiting the orthopedics department with non-osteoporotic fractures were excluded from the study. The patient data collection form was designed as per the need of the study. The patient data collection form includes demographic details of the patient, complaints on admission, medical and medication histories, personal history, social habits, vital signs, diagnosis, adverse drug reactions, laboratory parameters, and medication details. All the patients visiting the orthopedics department were reviewed on daily basis and those who met the study criteria were included in the study. All the relevant details including demographics, laboratory reports, type of fractures, and the prescribing pattern of the study populations have been documented in the data collection form. This was continued throughout the study period till the target sample size was achieved. The drug therapy of all the patients was analyzed with respect to the osteoporotic fractures, receiving calcium, Vitamin D, and anti-osteoporosis drugs. This was performed to assess the prescribing pattern of calcium, Vitamin D supplements, and anti-osteoporosis drugs and also the comorbidities and their treatment and the most common type of osteoporotic fractures.

The data collected were compiled, tabulated in Microsoft Excel 2013, and finally analyzed using statistical package for social science version 16.0 for windows. Quantitative variables were described as mean $\pm \mathrm{SD}$, and categorical variables were described as frequencies and percentages.

\section{RESULTS}

A total of 73 patients' prescriptions were reviewed during the study period. Considering the gender wise distribution of the study population, female predominance $(54.8 \%[\mathrm{n}=40])$ was noted over males $(45.2 \%$ [ $n=33])$. The average age of the patient was $63.52 \pm 11.06$ (mean \pm SD) years (range: 50 -88 years). The age-wise categorization of study population shows that $50 \%$ of the study population belongs to the age group of 50-60 years followed by $23 \%$ of age between 61 and 70 years. The distribution of study population is shown in Table 1.

\section{Pattern of comorbidities}

A total of 81 comorbidities were identified from 73 patients during the study period. Among them, 27 (36.98\%) patients had one comorbidity, $21(28.76 \%)$ patients had two comorbidities, $11(15.06 \%)$ patients had three or more comorbidities, and $14(19.17 \%)$ patients had no comorbidities. Considering the pattern of comorbidities among the study population, it was found that hypertension $(27.16 \%)$ was the most frequently occurring comorbidities followed by diabetes mellitus (18.51\%) and coronary artery diseases (14.81\%). The pattern of comorbidities among the study population is shown in Fig. 1.

\section{Types of fractures}

The types of fractures observed were metaphyseal fractures in $61.64 \%$ of the patients and diaphyseal fractures in $23.28 \%$ of the patients. The other fractures observed in the patients include mandibular angle fracture $(1.36 \%)$, spine compression fracture $(2.72 \%)$, Colles' fracture of wrist $(2.72 \%)$, patella fracture $(2.72 \%)$, calcaneus fracture $(4.08 \%)$, and compression fracture of dorsal spine (1.36\%).

\section{Classification of drugs prescribed}

A total of 709 drugs belonging to different classes were prescribed during the study period. Nonsteroidal anti-inflammatory drugs (NSAIDs) (17.06\%) were the most commonly prescribed class of drugs followed by antiulcer drugs (14.95\%), opioid analgesics (11.56\%), and anti-infective agents (11.14\%). The various class of drugs prescribed in patients with osteoporotic fractures is shown in Table 2.

\section{Distribution of calcium and Vitamin D supplementation}

Considering the prescribing pattern of calcium and Vitamin D supplementation among the study subjects, it was found that majority
Table 1: Demographic details of the study populations with osteoporotic fractures

\begin{tabular}{lll}
\hline S. No. & Characteristics & $\mathbf{n = 7 3}(\mathbf{\% )}$ \\
\hline 1 & Gender & \\
& Male & $33(45.2)$ \\
& Female & $40(54.8)$ \\
& Age group (y) & \\
& $50-60$ & $37(50.68)$ \\
& $61-70$ & $17(23.29)$ \\
& $71-80$ & $15(20.55)$ \\
& $\geq 81$ & $04(5.48)$ \\
\hline
\end{tabular}

Table 2: Drugs coprescribed in osteoporotic fractures

\begin{tabular}{lll}
\hline S. No & Drugs & $\mathbf{n = 7 0 9}(\%)$ \\
\hline 1 & NSAIDs & $121(17.06)$ \\
2 & Antiulcer drugs & $106(14.95)$ \\
3 & Opioid analgesics & $82(11.56)$ \\
4 & Anti-infective agents & $79(11.14)$ \\
5 & Vitamins & $75(10.57)$ \\
6 & Vitamin D granules & $58(8.18)$ \\
7 & Pregabalin & $46(6.48)$ \\
8 & Calcium supplements & $44(6.20)$ \\
9 & Antihypertensive agents & $32(4.51)$ \\
10 & Antidiabetic agents & $29(4.09)$ \\
11 & Cardiovascular agents & $16(2.25)$ \\
12 & Antiasthma drugs & $13(1.83)$ \\
13 & Steroids & $6(0.84)$ \\
14 & Bisphosphonates & $2(0.28)$ \\
\hline
\end{tabular}

NSAIDs: Nonsteroidal anti-inflammatory drugs

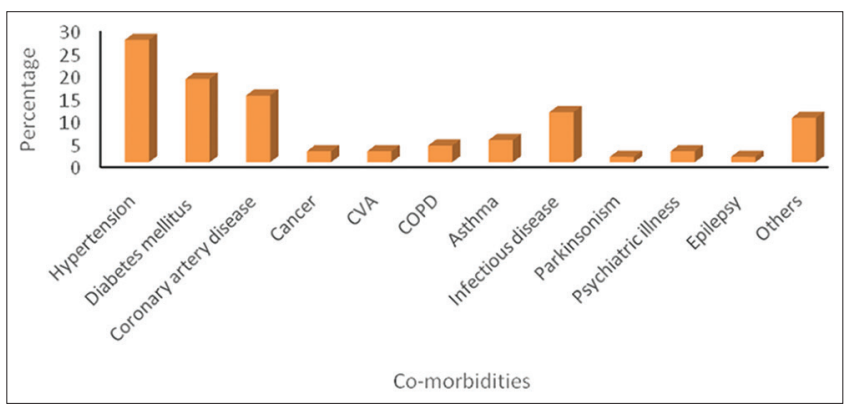

Fig. 1: Comorbidities among the study population

of patients $(65.75 \%)$ received both calcium and Vitamin D3 in their prescription. The anti-osteoporosis drug bisphosphonate was prescribed only in $2.73 \%$ of the patients and the remaining $31.5 \%$ of patients were prescribed with neither calcium, Vitamin D, nor bisphosphonate in their prescription. The prescribing pattern of calcium and Vitamin D3 along with bisphosphonates is shown in Fig. 2.

\section{Dosing of supplemental calcium and Vitamin D supplements}

Of the total study population who were prescribed with calcium supplementation, $82.92 \%$ were taking $<500 \mathrm{mg}$ of calcium daily, $14.63 \%$ taking $500-1000 \mathrm{mg}$, and $2.43 \%$ taking $>1000 \mathrm{mg}$ of calcium. In case of Vitamin D supplementation, $63.01 \%$ of patients were prescribed with $0.25 \mu \mathrm{g}, 26.02 \%$ were prescribed with $60,000 \mathrm{IU}$, and $1.36 \%$ were prescribed with 250IU of Vitamin D supplementation. Dosing of Vitamin D3 among the study population is shown in Table 3.

\section{DISCUSSION}

Osteoporotic fractures are frequent in elderly populations, especially in women and are associated with increased morbidity and mortality with frequent hospitalization and high health-care expenditure. Hence, there is an increased need of studying the different aspects related to bone health. For doing so, we have to determine the risk factors associated, 
comorbidities involved, and the drug therapy required for prevention and treatment $[9,10]$.

The study result showed that majority of the patients with osteoporotic fracture in the age group of 50-60 years with a mean age of $63.52 \pm 11.06$ years. This can be due to the progression of bone degradation due to aging as a consequence of higher bone turnover rate. Furthermore, the gender distribution showed that females were more prone to osteoporotic fractures than male patients. Similar results were seen in a study where the majority of patients were women and mean age was 67.8 years [11]. This might be due to menopause which leads to estrogen deficiency causing bone loss and calcium excretion. The cause of bone resorption in males can be explained by the decrease in testosterone levels [12].

Majority of the patients in our study showed a predominance of femur fractures as compared to other fractures. These findings were similar to the previous studies carried out on calcium intake in elderly patients with osteoporotic fractures which showed higher incidence of femur fractures $[13,14]$.

Various studies have shown that there is an association between comorbidities and the risk of fracture with the most prevalent comorbidities as diabetes and cardiovascular diseases $[15,16]$. In our study, hypertension, coronary artery disease, and diabetes were found to be commonly occurring comorbidities. These comorbid conditions are more prevalent in the higher aged individuals, and it interferes with the repair mechanism of the body. Furthermore, the drug therapy requirements increase along with the other existing diseases. This can also prolong the hospitalization, negatively increase the adverse effects and increase the financial burden on patients. Because of the increased number of drug prescriptions, there may be drug related problems as well as diminished efficacy of the drugs used for fracture treatment. This significantly affects the quality of life of the patients.

Global strategies for the prevention of fractures involve measures such as regular exercise, hip protectors, smoking cessation, reducing alcohol consumption, and diet modification. Daily dietary intake of calcium and Vitamin D is an important factor in the prevention and treatment of osteoporotic fractures. Daily intake of foods which are rich in calcium

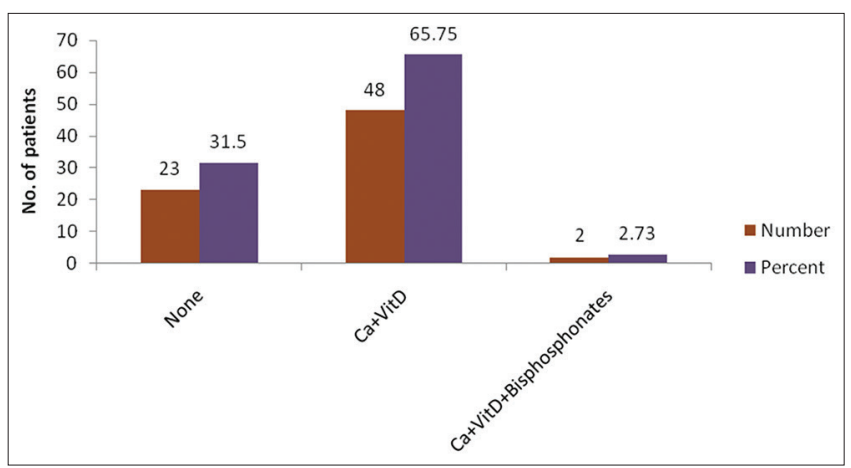

Fig. 2: Prescribing pattern of calcium, Vitamin D3, and bisphosphonates and Vitamin D such as kale, spinach, broccoli, okra, soya bean, sardines, salmon, egg yolk, fortified dairy products, orange, and oatmeal is necessary to improve bone strength [17]. Selection of an optimal therapy proves to be challenging due to the coexisting conditions and their treatment. The mainstay of therapy in osteoporotic fracture patients is supplementation with calcium and Vitamin D. Calcium plays an essential role in maintaining bone health across all ages and insufficient intake of calcium negatively affects bone metabolism and Vitamin D helps in absorbing the required amount of calcium. Bisphosphonates have been proved to be effective in preventing the fractures in patients with risk but are not generally used [18].

Calcium and Vitamin D supplementation were prescribed more in the patients aged between 50 and 60 years. The prescribed doses of calcium and Vitamin D were lower than that recommended in various literatures $[19,20]$. Most of the calcium and Vitamin D preparations prescribed were not generic which also adds to the financial burden.

Apart from fracture treatment, it is essential to manage the underlying disease and patients' health status. For pain management, it was noted that majority of the patients were prescribed with NSAIDs and opioid analgesics. These findings were similar to the results of a previous study which showed that NSAIDs were prescribed in $97 \%$ of the patients [21]. Paracetamol and diclofenac were the most commonly used painkillers for the treatment of mild-moderate pain, and tramadol was used for severe pain. Furthermore, anti-inflammatory enzymes, i.e., trypsin and chymotrypsin with or without diclofenac were found to be used in most of the patients. In our study, most of the patients received antibiotics for the management of post-operative fracture-associated infections. Among the antibiotics prescribed, the third-generation cephalosporins were given more frequently. The choice of antibiotics must be based on the most likely pathogen at the site of the infection, and it is always better to carry out the culture/sensitivity tests so as to prevent the irrational use of antibiotics and the emergence of drug resistance.

Majority of the prescriptions were coprescribed with proton-pump inhibitors (PPIs) (80.82\%) (Pantoprazole) as compared to histamine-2 receptor antagonists $\left(\mathrm{H}_{2} \mathrm{RA}\right)$ (ranitidine) to minimize drug induced gastritis associated with antibiotics, NSAIDs, and opioid analgesics. Our study results were inconsistent with other studies which showed that $\mathrm{H}_{2} \mathrm{RA}$ were the most commonly coprescribed to minimize drug-induced gastritis [22,23].

Even though PPIs produce more sustained acid suppression and promote ulcer healing, substitution with other gastrointestinal protective agents such as antacids and ranitidine may potentially yield substantial cost savings. The patient education and knowledge about medicines will help to improve adherence and is considered vital for preventing and management of osteoporosis-related fractures.

\section{CONCLUSION}

The study shows the pattern of different types of osteoporotic fractures in geriatric patients and the drug use among them. Considering that calcium and Vitamin D supplementation are essential for the treatment of osteoporosis, regular monitoring of serum calcium and Vitamin D3 levels should be put into health-care practice more widely so that

Table 3: Dosing of calcium and vitamin D in study populations

\begin{tabular}{|c|c|c|c|c|c|c|}
\hline \multirow[t]{3}{*}{ Age group (y) } & \multicolumn{6}{|c|}{ Number of patients } \\
\hline & \multicolumn{3}{|c|}{ Calcium (mg/day) } & \multicolumn{3}{|l|}{ Vitamin D } \\
\hline & $<500$ & $500-1000$ & $>1000$ & $0.25 \mu \mathrm{g} / \mathrm{day}$ & 250 IU/day & $60 \mathrm{kIU} /$ week \\
\hline $50-60$ & 16 & 3 & 0 & 24 & 0 & 7 \\
\hline $60-70$ & 12 & 1 & 0 & 13 & 0 & 5 \\
\hline $70-80$ & 3 & 1 & 1 & 5 & 1 & 5 \\
\hline $80-90$ & 3 & 1 & 0 & 4 & 0 & 2 \\
\hline
\end{tabular}


the daily dose of calcium and Vitamin D3 can be tailored according to the individual needs. The patient education lifestyle and dietary supplements play a very important role in the management of osteoporosis; further, efforts should be taken to increase the adherence of calcium, particularly in osteoporotic fractures.

\section{ACKNOWLEDGMENT}

We would like to express our gratitude to the head of the Orthopaedics Department and medical superintendent of Justice K S Hegde Charitable Hospital, Deralakatte, Mangaluru, for giving permission to conduct the study. Heartfelt thanks to the principal, NGSM Institute of Pharmaceutical Sciences, for providing necessary support and encouragement to the study. We also take this opportunity to express our thanks to those who have directly and indirectly guided us, for their immense support that helped us to complete this work.

\section{AUTHORS' CONTRIBUTION}

All authors listed, have made substantial, direct and intellectual contribution to the work, and approved it for publication.

\section{CONFLICTS OF INTEREST}

The authors declare that they have no conflicts of interest.

\section{REFERENCES}

1. Jeon YK, Kim BH, Kim IJ. The diagnosis of osteoporosis. J Korean Med Assoc 2016;59:842-6.

2. Tavakoli-Ardakani M, Eshraghi A, Hajhossein Talasaz A, Salamzadeh J. A drug utilization evaluation study of amphotericin B in neutropenic patients in a teaching hospital in Iran. Iran J Pharm Res 2012;11:151-6.

3. Shah RB, Gajjar BM, Desai SV. A study on drug utilization pattern among geriatric patients assessed with anatomical therapeutic chemical classification/defined daily dose system in rural tertiary care teaching hospital. Int J Nutr Pharm Neurol Dis 2012;2:258-65.

4. Jackson RD, LaCroix AZ, Gass M, Wallace RB, Robbins J, Lewis CE, et al. Calcium plus vitamin D supplementation and the risk of fractures. N Engl J Med 2006;354:669-83.

5. Chapuy MC, Pamphile R, Paris E, Kempf C, Schlichting M, Arnaud S, et al. Combined calcium and vitamin D3 supplementation in elderly women: Confirmation of reversal of secondary hyperparathyroidism and hip fracture risk: The decalyos II study. Osteoporos Int 2002;13:257-64.

6. Cosman F, de Beur SJ, LeBoff MS, Lewiecki EM, Tanner B, Randall S, et al. Clinician's guide to prevention and treatment of osteoporosis. Osteoporos Int 2014;25:2359-81

7. Weycker D, Li X, Barron R, Bornheimer R, Chandler D. Hospitalizations for osteoporosis-related fractures: Economic costs and clinical outcomes. Bone Rep 2016;5:186-91.
8. Zam W. Vitamin D deficiency and depressive disorders: Review study of probable relationship. Int J Pharm Pharm Sci 2016;8:16-22.

9. Warensjö E, Byberg L, Melhus H, Gedeborg R, Mallmin H, Wolk A, et al. Dietary calcium intake and risk of fracture and osteoporosis: Prospective longitudinal cohort study. BMJ 2011;342:d1473.

10. Tripathy A, Adiga S, Shah HH, Shanbhag TV, Kumar MD. A retrospective study of clinical profile and drug prescribing pattern in osteoporosis in a tertiary care teaching hospital. Int J Pharm Pharm Sci 2015;7:390-3.

11. Tang BM, Eslick GD, Nowson C, Smith C, Bensoussan A. Use of calcium or calcium in combination with vitamin $\mathrm{D}$ supplementation to prevent fractures and bone loss in people aged 50 years and older: A meta-analysis. Lancet 2007;370:657-66.

12. O'Connell MB, Borchert SS. Osteoporosis and other metabolic bone diseases. In: Dipiro JT, Talbert RL, Yee GC, Matzke GR, Wells BG, Posey LM, editors. Pharmacotherapy: A Pathophysiological Approach. $9^{\text {th }}$ ed. USA: Mc Graw-Hill Education; 2014. p. 1482-3.

13. Cho K, Cederholm T, Lökk J. Calcium intake in elderly patients with hip fractures. Food Nutr Res 2008;52:1-5.

14. Yoon DS, Lee YK, Ha YC, Kim HY. Inadequate dietary calcium and Vitamin D intake in patients with osteoporotic fracture. J Bone Metab 2016;23:55-61.

15. Sugimoto T, Sato M, Dehle FC, Brnabic AJ, Weston A, Burge R, et al. Lifestyle-related metabolic disorders, osteoporosis, and fracture risk in Asia: A Systematic review. Value Health Reg Issues 2016;9:49-56.

16. McFarlane SI, Muniyappa R, Shin JJ, Bahtiyar G, Sowers JR. Osteoporosis and cardiovascular disease: Brittle bones and boned arteries, is there a link? Endocrine 2004;23:1-0.

17. Food Sources of Calcium and Vitamin D; 2017. Available from: http:// www.healthlinkbc.Ca/sources-calcium-vitamin-D

18. Kannegaard PN, van der Mark S, Eiken P, Abrahamsen B. Excess mortality in men compared with women following a hip fracture. National analysis of comedications, comorbidity and survival. Age Ageing 2010;39:203-9.

19. Kim SH, Ko YB, Lee YK, Hong SW, Choi HJ, Ha YC, et al. National utilization of calcium supplements in patients with osteoporotic hip fracture in Korea. J Bone Metab 2013;20:99-103.

20. Castro-Lionard K, Dargent-Molina P, Fermanian C, Gonthier R, Cassou B. Use of calcium supplements, vitamin D supplements and specific osteoporosis drugs among French women aged 75-85 years: Patterns of use and associated factors. Drugs Aging 2013;30:1029-38.

21. Patel NS, Shah RB, Buch JG. Study on drug utilization pattern of nonsteroidal anti-inflammatory drugs assessed with anatomical therapeutic chemical classification/defined daily dose system in an orthopedics department. Drug Ther Perspect 2015;31:358-64.

22. Shehnaz SI, Arifulla M, Sreedharan J, Sam KG. Prescribing patterns in orthopedics outpatient department in GMC Hospital, Ajman, UAE. Gulf Med J ASM 2014;3:S120-31.

23. Kanagasanthosh K, Topno I. A study on recent trends of prescribing pattern in orthopedics out-patient department from a tertiary care teaching hospital. Int J Pharm Technol 2015;6:7549-59. 